\title{
Multimodal Imaging of Alzheimer Pathophysiology in the Brain's Default Mode Network
}

\author{
Jonghan Shin,, ${ }^{1,2,3}$ Vladimir Kepe, ${ }^{3}$ Gary W. Small, ${ }^{2}$ Michael E. Phelps, ${ }^{3}$ and Jorge R. Barrio ${ }^{3}$ \\ ${ }^{1}$ Neuroscience Research Institute, Gachon University of Medicine and Science, Incheon 405-760, Republic of Korea \\ ${ }^{2}$ Department of Psychiatry and Biobehavioral Sciences and Semel Institute for Neuroscience and Human Behavior, The University of \\ California, Los Angeles David Geffen School of Medicine, Los Angeles, CA 90095-6948, USA \\ ${ }^{3}$ Department of Molecular and Medical Pharmacology, The University of California, Los Angeles David Geffen School of Medicine, \\ Los Angeles, CA 90095-6948, USA
}

Correspondence should be addressed to Jonghan Shin, jhshin@gachon.ac.kr

Received 5 December 2010; Accepted 31 January 2011

Academic Editor: Akihiko Takashima

Copyright (๑) 2011 Jonghan Shin et al. This is an open access article distributed under the Creative Commons Attribution License, which permits unrestricted use, distribution, and reproduction in any medium, provided the original work is properly cited.

\begin{abstract}
The spatial correlations between the brain's default mode network (DMN) and the brain regions known to develop pathophysiology in Alzheimer's disease $(\mathrm{AD})$ have recently attracted much attention. In this paper, we compare results of different functional and structural imaging modalities, including MRI and PET, and highlight different patterns of anomalies observed within the DMN. Multitracer PET imaging in subjects with and without dementia has demonstrated that [C-11]PIB- and [F18]FDDNP-binding patterns in patients with $\mathrm{AD}$ overlap within nodes of the brain's default network including the prefrontal, lateral parietal, lateral temporal, and posterior cingulate cortices, with the exception of the medial temporal cortex (especially, the hippocampus) where significant discrepancy between increased [F-18]FDDNP binding and negligible [C-11] PIB-binding was observed. [F-18]FDDNP binding in the medial temporal cortex — a key constituent of the DMN—coincides with both the presence of amyloid and tau pathology, and also with cortical areas with maximal atrophy as demonstrated by T1-weighted MR imaging of AD patients.
\end{abstract}

\section{Introduction}

In 2001, the default mode network (DMN) was first proposed as an interconnected set of brain regions that is active when the brain is in a resting state and typically deactivated during memory encoding and other cognitively demanding tasks focused on processing of external stimuli [1]. The DMN includes the dorsal and ventral medial prefrontal cortices, medial and lateral parietal cortex, and parts of the medial and lateral temporal cortices.

It has been suggested that the DMN consists of two subsystems that converge on two core regions: anterior medial prefrontal cortex and posterior cingulate cortex. The two subsystems, dorsal medial prefrontal cortex subsystem (consisting of dorsal medial prefrontal cortex, temporoparietal junction, lateral temporal cortex, and temporal pole) and medial temporal cortical subsystem (consisting of ventral medial prefrontal cortex, posterior inferior parietal lobule, retrosplenial cortex, parahippocampal cortex, and hippocampal formation) may be activated simultaneously or separately during these tasks [2]. Tasks during which the DMN is activated include, among others, autobiographical memory, thinking about one's future, theory of mind, selfreferential and affective decision-making [2].

Alzheimer's disease (AD) affects many aspects of cognitive function, in particular memory, attention, visuospatial orientation, and language. These changes in multiple domains point to widespread abnormalities in brain circuitry at more advanced stages of disease [3]. Early symptomatology of $\mathrm{AD}$ has been linked to changes in the default mode network based on glucose metabolism and blood flow PET and SPECT measurements and later with fMRI experiments (reviewed by [4]). As a result, the default network rapidly became the target of extensive research in the AD field [49]. In addition to extensive cortical neuronal loss, $\mathrm{AD}$ is also defined by the presence of brain amyloid pathology (amyloid 
plaques) and tau pathology (neurofibrillary tangles) [10, 11], postmortem detection of which is required for the definitive diagnosis of $\mathrm{AD}$.

In this paper, we compare results of different functional and structural imaging modalities used in $\mathrm{AD}$ imaging including T1-wighted MR imaging, fMRI, 2-deoxy-2-[F18]fluoro-D-glucose ([F-18]FDG)-PET, 6-hydroxy-2-(4' ([C-11]methylamino)phenyl)benzothiazole ([C-11]PIB)PET, and 2-(1-\{6-[(2-[F-18]fluoroethyl)(methyl)amino]2-naphthyl ethylidene)malononitrile ([F-18]FDDNP)-PET imaging from the perspective of the brain's DMN. Imaging results across imaging modalities probing different pathophysiological changes in AD show different patterns of anomalies observed within the DMN. For example, declines in [F-18]FDG PET measures of glucose metabolism in posterior cingulate gyrus at the earliest presymptomatic stages of $\mathrm{AD}$ and progression to parietal and lateral temporal cortices in mild $\mathrm{AD}$ [12] were early observed, yet these functional changes cannot be attributed only to cortical atrophy in these regions, but also to deficits in neuronal projections resulting from pathology deposition and neuronal degeneration in the medial temporal cortex [13]. This is also shown in the effect of entorhinal and rhinal lesioning on posterior cingulate regional cerebral glucose utilization in baboons [14]. Robust atrophy as well as significantly increased [F-18]FDDNP binding was found within the medial temporal cortex and other parietal and temporal regions at early stages of AD [15-18]. [F-18]FDDNP is the first PET molecular imaging probe successfully applied for in vivo visualization of Alzheimer's disease pathology in the brain of living AD patients with a pattern of increased binding in the brain areas known to contain both amyloid plaques and neurofibrillary tangles $[13,16,19,20]$. These brain areas include medial temporal and lateral temporal cortices, posterior cingulate gyrus, parietal and frontal cortices, all of which are involved in the DMN. Similarly several studies have correlated [C-11]PIB binding with the anatomical substrates of the DMN $[4,21-$ $23]$ and have found increased [C-11] PIB binding in several, but not all, DMN brain regions. Recently, multitracer PET imaging using both [F-18]FDDNP and [C-11]PIB in the same subjects with and without dementia have provided important information on clear and meaningful similarities and differences in topographical distribution between [C-11]PIB-PET and [F-18]FDDNP-PET images [24-27].

\section{Emergence of the Default Mode Network}

Raichle, Gusnard, and colleagues introduced the concept of DMN with three fMRI papers in 2001 [1, 28, 29]. Their papers directly considered the empirical and theoretical implications of defining baseline states and what the specific pattern of activity in the default mode network might represent [4]. Prior to the availability of fMRI the activation studies using [O-15] $\mathrm{H}_{2} \mathrm{O}$ PET (and other perfusion agents) have been used to study activity patterns of the brain in the resting state. Figure 1 shows the common, default mode activity in young adults measured by $[\mathrm{O}-15] \mathrm{H}_{2} \mathrm{O}$ PET (see

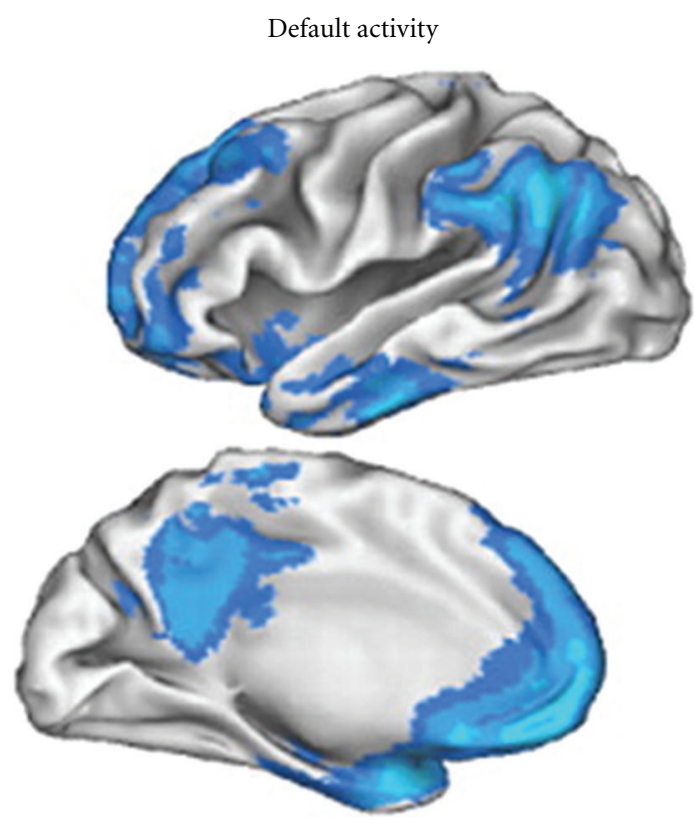

FIGURE 1: The brain's default network was originally identified in a meta-analysis that mapped brain regions more active in passive as compared to active tasks (often referred to as task-induced deactivation). The displayed $[\mathrm{O}-15] \mathrm{H}_{2} \mathrm{O}$ positron emission tomography (PET) data include nine studies (132 young adults) from Shulman et al. [30] (reanalyzed in [21]). Images show the medial and lateral surface of the left hemisphere using a population-averaged surface representation to take into account between-subject variability in sulcal anatomy. Blue represents regions most active in passive task settings. Adapted and reprinted with permission from Buckner et al. $[4,21]$ [Copyright (2005) Journal of Neuroscience].

$[4,21,30]$ for detail). These results point to a wide network of regions activated in the default state and includes the precuneus (the portion of the parietal cortex caudal to the paracentral lobule) extending into posterior cingulate and retrosplenial cortex, as well as inferior lateral parietal regions, frontal regions along the midline, medial, and temporal cortex $[4,21,31-33]$.

\section{Disruption of the Default Network in AD}

3.1. MRI Measures of Atrophy. A variety of pathological processes including neuronal loss, synaptic loss, and loss of projections contribute to gray matter atrophy which spreads across the brain in $\mathrm{AD}$ with the disease progression disrupting neuronal circuits involved in the DMN prominently including the medial temporal cortex [17, 21, 22, 34, 35]. The temporal trajectory of cortical atrophy was studied by Whitwell et al. [18] in a longitudinal MRI study of atrophy in amnestic MCI (aMCI) subjects who progressed to AD. The initial pattern of grey matter loss in the aMCI subject scans 3 years prior to the diagnosis of $\mathrm{AD}$ was focused primarily in the medial temporal cortex, including the amygdala, anterior hippocampus and entorhinal cortex, with some additional involvement of the fusiform gyrus,. The extent and magnitude of cerebral atrophy in these patients further progressed 

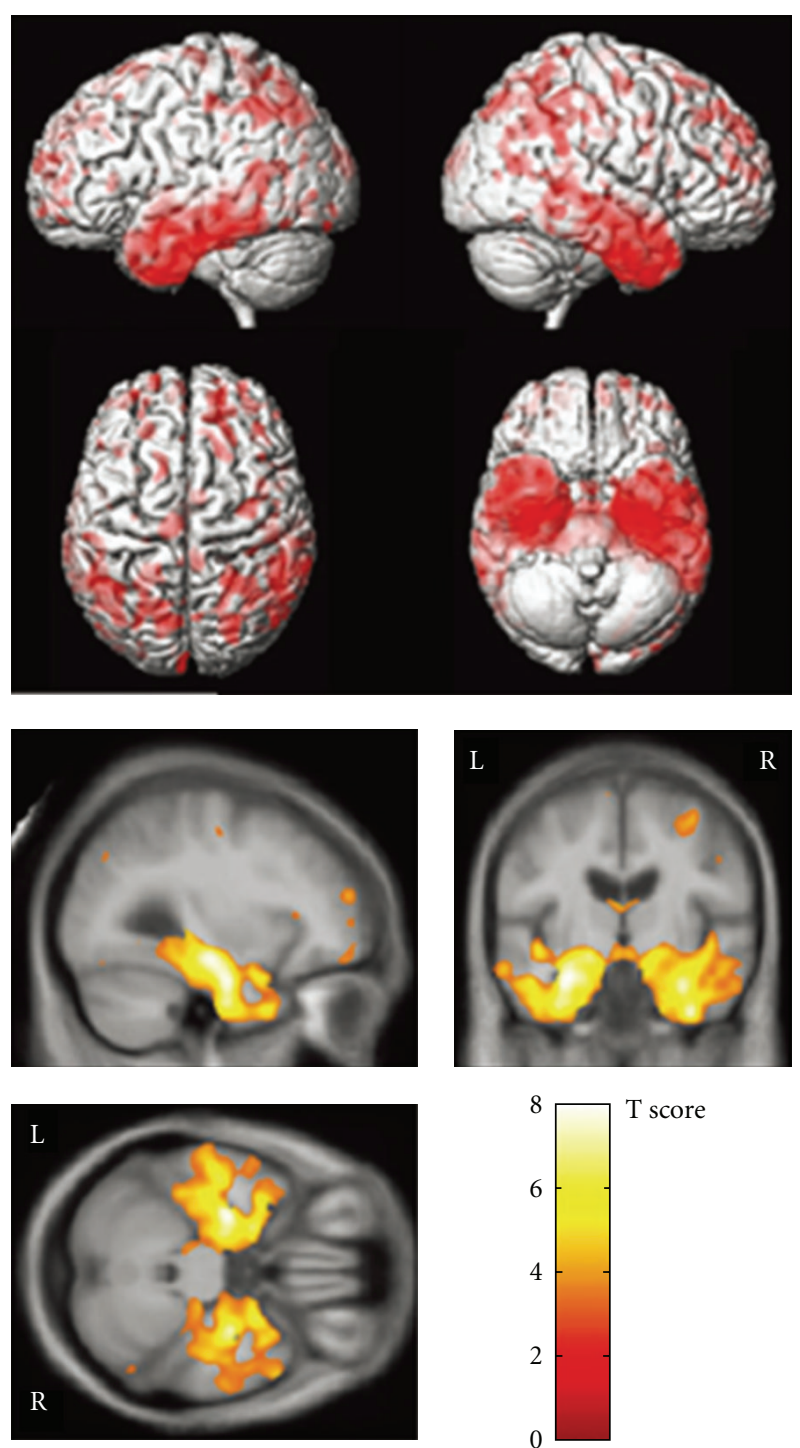

Figure 2: Patterns of grey matter atrophy in patients with $\mathrm{AD}$ compared with age-matched control group. The results are shown on a $3 \mathrm{D}$ surface render (top) and overlaid on representative axial, coronal and sagittal slices (bottom). L: left; R: right. Adapted and reprinted with permission from Whitwell et al. [18] [Copyright (2005) Brain].

over the following two years with atrophy in the medial temporal cortex spreading to middle temporal gyrus, and extended into more posterior regions of the temporal cortex to include the entire extent of the hippocampus and partial involvement of the parietal cortex. By the time the subjects had progressed to a clinical diagnosis of $\mathrm{AD}$ the pattern of grey matter atrophy had become more widespread with more severe involvement of the medial temporal cortex and the temporoparietal association cortices and, for the first time, substantial involvement of the frontal cortex (see Figure 2).

Accelerated atrophy is also observed in the posterior cingulate gyrus, one of the core regions of DMN [2] and an area with the earliest decline in glucose utilization in the preclinical stages of $\mathrm{AD}$ [12]. This implies that the early functional abnormalities and structural changes of neuronal circuitry in the default mode network only involve a subset of DMN core regions $[4,21]$ to progress later to other areas of the network.

3.2. Functional MRI. Recently, functional changes in the default mode network have been explored with AMRI in AD using both analysis of task-induced deactivation [36, 37] and analysis of intrinsic activity correlations [33, 37-39].

Thus, by all measures the default mode network appears disrupted in $\mathrm{AD}$, including prominently the medial temporal cortex subsystem and posterior cingulate area [4].

\section{PET Imaging of Amyloidosis and Tauopathy in AD}

4.1. [F-18]FDDNP-PET in AD. Beta-amyloid plaques and neurofibrillary tangles are the two most common neuropathological hallmarks of Alzheimer's disease and they appear in distinctive progressive temporal and spatial patterns of cortical pathology evolution during the disease progression [11]. [F-18]FDDNP is known to bind to amyloid plaques as well as neurofibrillary tangles in vitro [20]. Post mortem neuropathological studies of AD patients who previously received [F-18]FDDNP-PET scans show close matching of in vitro immunohistochemical determined pattern of plaque and tangle distribution in the brain regions with imaging results [16]. [F-18]FDDNP PET-binding patterns of subjects with mild cognitive impairment and AD patients [16] is progressive. Initially increased medial temporal cortex binding in controls at risk and MCI subjects is followed by a variable and progressive pattern of binding in one or more of other cortical areas including posterior cingulate gyrus. In contrast, more advanced $\mathrm{AD}$ patients had a pattern of elevated [F-18]FDDNP binding in prefrontal, parietal, posterior cingulate, lateral temporal and medial temporal cortices areas $[15,16,24]$. These results in living humans clearly point at the medial temporal cortex as being the area with the earliest pathological lesion formation which is in accordance with neuropathology findings [11]. Figure 3 shows three-dimensional cortical surface projection images of [F-18]FDDNP-PET scans from a representative patient with AD.

4.2. [C-11]PIB-PET in AD. PET imaging studies with [C11]PIB, which has been suggested to bind to amyloid plaques only $[40,41]$, show a single pattern of [C-11]PIB binding in the prefrontal, lateral parietal, posterior cingulate, lateral temporal cortices and precuneus [40] at all levels of cognitive impairment, including AD, a subset of MCI subjects and even some cognitively normal controls. This pattern of [C11]PIB cortical uptake was correlated with the DMN by Buckner et al. [21] who noticed overlap of DMN and [C11]PIB-binding pattern in cortical areas with exception of the medial temporal cortex which include both hippocampus and parahippocampal gyrus (see Figure 4). When compared with the patterns of known beta-amyloid deposition in $\mathrm{AD}[11,42,43]$, this lack of [C-11]PIB binding in the 


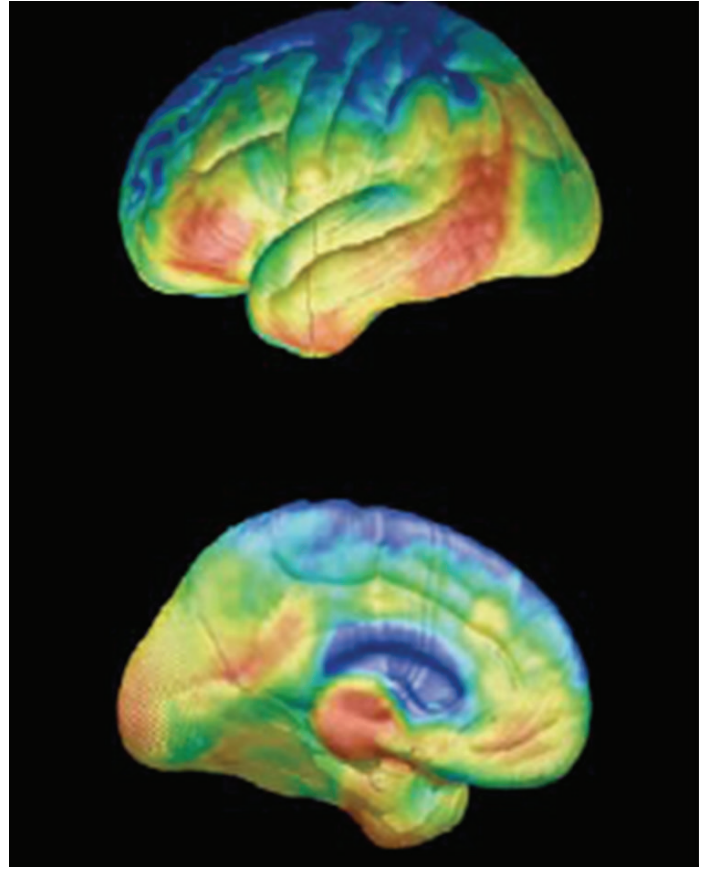

FIgURE 3: Three-dimensional cortical surface projection images of [F-18]FDDNP-PET scans from a patient with AD. Lateral (upper) and medial (lower) brain surfaces are shown. Warmer colors indicate higher numbers of plaques and tangles. Adapted and reprinted with permission from Small et al. [16] [Copyright (2008) Lancet Neurology].

hippocampus and neocortical areas of medial temporal cortex (entorhinal, parahippocampal, rhinal, and perirhinal cortices) points to the most significant mismatch between beta-amyloid pathology and [C-11]PIB binding. Indeed multiple contradictory [C-11]PIB-binding results in humans have been reported [44] and recent work demonstrating that [C-11]PIB brain accumulation may be at least in part mediated by [C-11]PIB sulfation via estrogen sulfotransferase [45] has cast doubts on the purported human amyloid specificity of [C-11]PIB.

It may be possible that other factors, like cortical atrophy and/or low distribution of amyloid aggregates may contribute to the underestimation of [C-11]PIB accumulation in medial temporal lobe (MTL). Indeed, topographical differences in the distribution of senile plaques (SP) and neurofibrillary tangles (NFT) have been noted in $\mathrm{AD}$, with lower densities of SP in limbic areas of MTL, such as hippocampus and subiculum, which are more affected by neurofibrillary tangle pathology [10]. Yet in other areas of medial temporal lobe, such as entorhinal, perirhinal, and rhinal cortices as well as parahippocampal gyrus, $\beta$-amyloid plaque densities (both diffuse and neuritic plaques) reach levels observed in lateral temporal lobe and other cortical areas even in subjects without clinically manifested dementia and only limited atrophy [43]. Thus, even though some partial volume effects are expected, it is unlikely that [C11]PIB negligible binding in MTL at all levels of cognitive impairment is entirely due to the combined effects of low

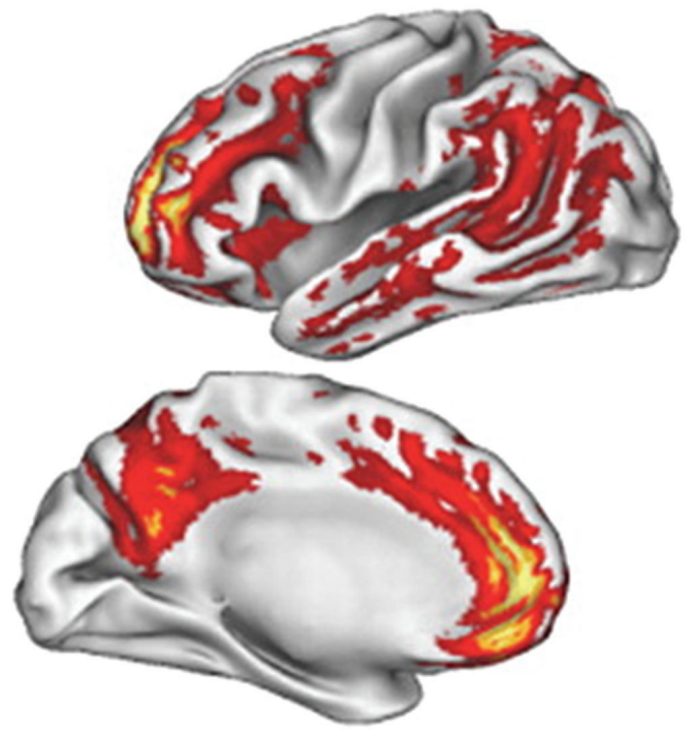

FIGURE 4: Surface maps indicating the pattern of [C-11]PIB binding obtained with 10 patients with clinical Alzheimer's disease compared with 29 healthy older control subjects. Adapted and reprinted with permission from Buckner et al. [21] [Copyright (2005) Journal of Neuroscience].

$\beta$-amyloid plaque densities and atrophy. If so, one would also expect a similar drastic effect of MTL atrophy with the [F-18]FDDNP signal in the same patients, and this is not observed (Figures 5 and 6 ).

\section{5. [F-18]FDDNP- and [C-11]PIB-PET Comparison in the Same AD Patients}

Postmortem pathological studies of AD brains demonstrated that the distribution of neuritic plaques may present significant intersubject variability. $[10,11]$. To minimize this effect and to compare more accurately binding patterns of both imaging probes, comparison of [F-18]FDDNP and [C-11]PIB binding in the same AD subjects is necessary. Recently, a comparative multitracer PET imaging study using both [F-18]FDDNP and [C-11]PIB has been performed in control subjects and patients with $\mathrm{AD}[24,25]$. These studies demonstrate that in $\mathrm{AD}$ both probes show widespread cortical uptake within the areas of the DMN including the prefrontal cortex, lateral parietal, lateral temporal cortex, and posterior cingulate cortices (Figure 5). However, these studies also demonstrate the critical difference between [C11]PIB and [F-18]FDDNP binding in the medial temporal cortex, where [F-18]FDDNP is high and [C-11]PIB binding is negligible in the same $\mathrm{AD}$ subjects.

To highlight the different binding pattern of [F18]FDDNP and [C-11]PIB in the medial temporal cortex, the subtracted PET imaging of [F-18]FDDNP minus [C11]PIB in the same subjects with $A D$ was calculated. Figure 6 demonstrates that the medial temporal cortex was the most significant differential brain region in the voxel mapping of the subtracted PET imaging. 


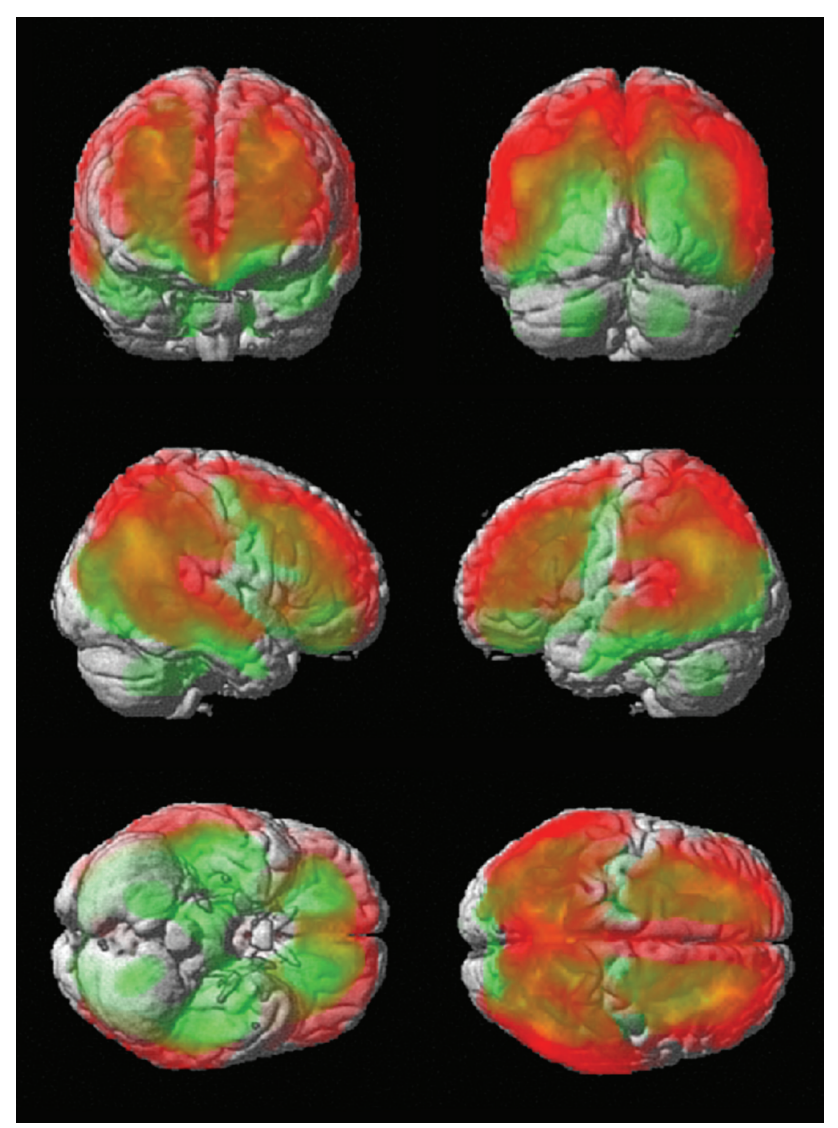

FIgURE 5: Comparison of [C-11]PIB SPM $t$-map image (red) and [F-18]FDDNP SPM $t$-map image (green) obtained with $10 \mathrm{AD}$ patients compared with 10 healthy age-matched control subjects $(P<.05$, uncorrected, $k=100)$. The yellow area represents the area where the [C-11]PIB SPM $t$-map (red) and [F-18]FDDNP SPM $t$ map (green) overlapped. Adapted and reprinted with permission from Shin et al. [25] [Copyright (2010) Neuroimage].

\section{Default Mode Network and Medial Temporal Cortex}

One has to approach with cautious interpretation of imaging data across the modalities in DMN since comparing activity with structural changes may lead to erroneous conclusions regarding $\mathrm{AD}$ and its progression. Resting state fMRI, [O15] $\mathrm{H}_{2} \mathrm{O}$ PET, and [F-18]FDG PET give us information about the activity of DMN during a variety of thought processes considered as resting state in healthy subjects and in $\mathrm{AD}$ patients. Functional changes in specific DMN nodes may result from the pathology present in the nodes themselves or can reflect pathological changes in connectivity of the circuits leading to and from the specific node. It is not surprising that neuropathological structural changes present at early stages of AD evolution (MRI atrophy in the medial temporal cortex) may not overlap with functional changes entirely ([F18]FDG decline in posterior cingulate gyrus). [F-18]FDG decline does reflect changes in functional activity of neuronal terminals (i.e., in posterior cingulate gyrus) caused by neuropathology in their cell bodies (i.e., in hippocampus and other medial temporal cortex substructures).
Comparison of [F-18]FDDNP and [C-11]PIB-binding patterns at early stages and late stages of $\mathrm{AD}$ shows stark difference between the immutable pattern of [C-11]PIBbinding patterns [46] and evolution of [F-18]FDDNPbinding with disease progression from initial localization in the medial temporal cortex to the widespread cortical pattern found in $\mathrm{AD}$ as expected from autopsy determinations. If one accepts the hypothesis that [C-11]PIB-binding reflects the level of beta-amyloid plaques in vivo then the obvious lack of [C-11]PIB-binding to the medial temporal cortex, an area with early and abundant deposition of amyloid plaques $[11,43]$ raises the question about the validity of this hypothesis. Although correlation of [C-11]PIB-binding pattern in $\mathrm{AD}$ and increased levels of regional consumption of glucose via glycolysis have been noted, there was poor correlation between the total level of glucose utilization (indicative of the remaining cell density and their functional activity in the affected areas) and the levels of [C11]PIB-binding. Longitudinal [C-11]PIB and [F-18]FDG PET studies in $\mathrm{AD}[47,48]$ have shown progressive cortical metabolic decline, as measured with [F-18]FDG, in parallel with cognitive function, but no significant changes in [C11]PIB binding levels or binding pattern over time were observed.

Early pathology observations have revealed that $\mathrm{AD}$ affects only a subset of neurons in regional and laminar specific neuronal systems [49] and that $\mathrm{AD}$ is a disconnection syndrome with damage to the entorhinal cortex and/or subiculum which effectively disconnects hippocampal formation from the rest of the neocortex. Subsequent progression of the disease occurs in a stepwise fashion along cortico-cortical connections [50]. Large cross-sectional studies have revealed that cortical neurofibrillary tangle densities and not amyloid deposits are the best correlates of the severity of dementia, neuronal loss, and atrophy [51].

These pathology observations support the hypothesis that $\mathrm{DMN}$ and its subsystems are affected early in $\mathrm{AD}$ with neurofibrillary tangles, neuronal loss, and atrophy in the medial temporal cortex and earliest metabolic changes in posterior cingulate area. Both areas are highly connected with other cortical and subcortical regions and are thus excellent starting points for spread of pathological changes as hypothesized by de Lacourte and White III [50].

\section{Conclusions}

The spatial correlations between the brain's default mode network and previously known $\mathrm{AD}$ pathophysiology patterns have become more evident with the advances in functional structural imaging techniques. Furthermore, development of PET molecular imaging probes also makes it possible to assess in vivo distribution of $\mathrm{AD}$ pathology within the default mode network activity in living AD patients. Atrophy measurements, [F-18]FDG PET and [F-18]FDDNP PET studies point to the medial temporal cortex and posterior cingulate gyrus as two areas of DMN with the earliest pathological and functional changes. Cross-sectional PET 

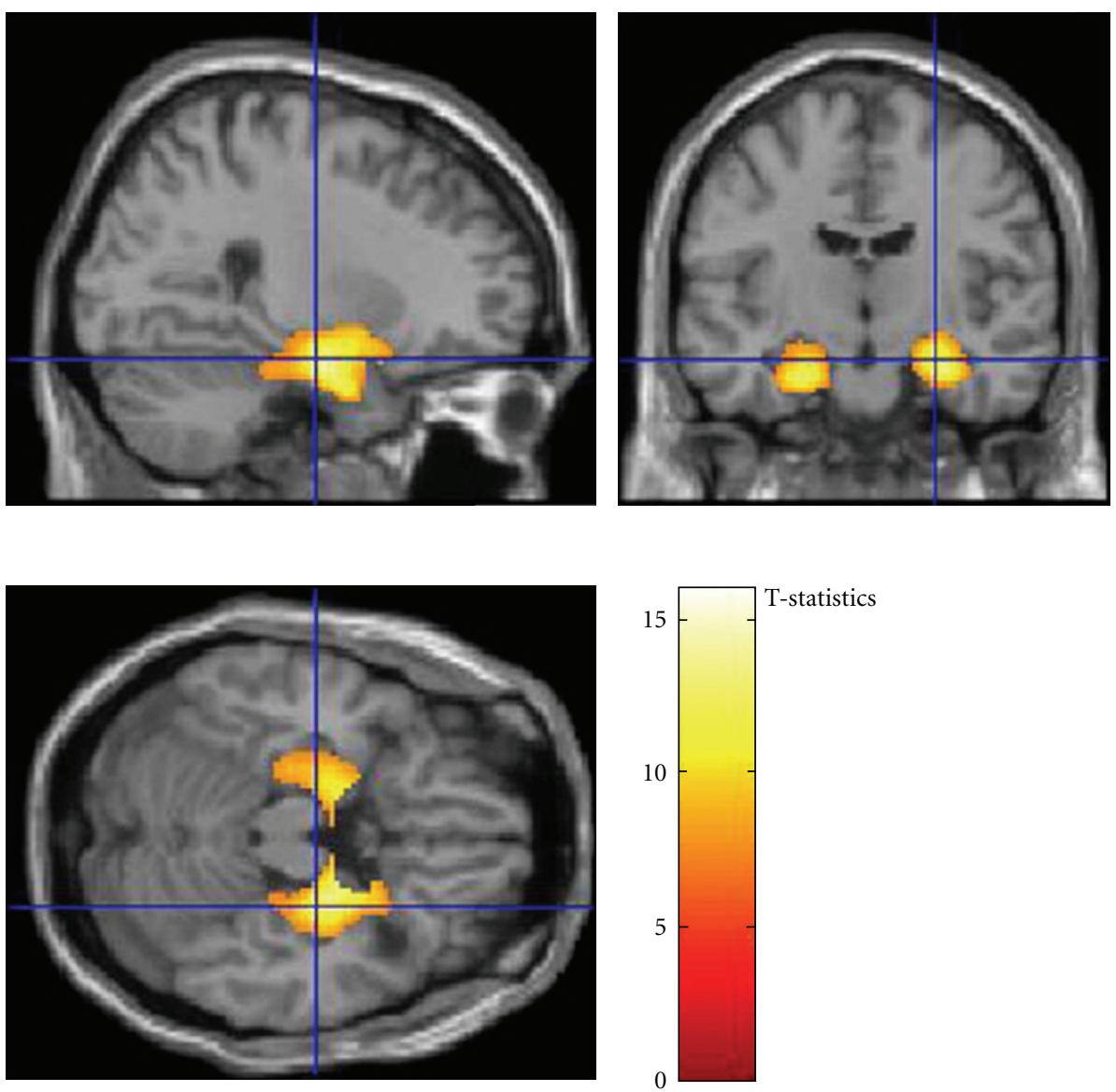

Figure 6: [F-18]FDDNP minus [C-11]PIB results (yellow blobs) in the same patients with AD as statistical parameter mapping (SPM) projections superimposed on a standardized magnetic resonance imaging brain template in the three orthogonal right sagittal (upper left), coronal (upper right), and axial (lower left) views $(P<.05$ (FWE); corrected, $k=100)$. Adapted and reprinted with permission from Shin et al. [25] [Copyright (2010) Neuroimage].

studies show initial [F-18]FDDNP binding in the medial temporal cortex and spreading stepwise to the other areas of DMN. [C-11] PIB determinations reveal a single pattern of binding within DMN which does not include the medial temporal cortex. This pattern is present in on-off fashion in control and MCI subjects [46]. Multitracer PET imaging determinations using both [F-18]FDDNP and [C-11]PIB in the same subjects, with and without $\mathrm{AD}$ showed overlaps between [C-11]PIB-binding patterns and [F-18]FDDNP binding patterns within the brain's default mode network. However, it also demonstrated a critical discrepancy found in the medial temporal cortex where [F-18]FDDNP binding is the highest but [C-11] PIB binding is negligible. Moreover, while [F-18] FDDNP shows the expected pattern of disease progression, [C-11]PIB shows uniform patterns of distribution throughout the DMN at various stages of disease evolution. Because marked AD pathology (demonstrated by postmortem histopathology) and atrophy (demonstrated by T1-weighted MR imaging) have their earliest manifestation in the medial temporal cortex, [C-11]PIB binding in the $\mathrm{DMN}$ is inconsistent with its purported specificity for betaamyloid aggregates.

\section{Acknowledgments}

This paper is supported by NIH Grants P01-AG024831 and DOE contract DE-FC03-87-ER60615. The University of California, Los Angeles, owns the U.S. patent $(6,274,119)$ entitled "Methods for Labeling Beta-Amyloid Plaques and Neurofibrillary Tangles." Drs. G. W. Small and J. R. Barrio are among the inventors. Dr. Barrio gratefully acknowledges the support of the Elizabeth and Thomas Plott Chair Endowment in Gerontology.

\section{References}

[1] D. A. Gusnard and M. E. Raichle, "Searching for a baseline: functional imaging and the resting human brain," Nature Reviews Neuroscience, vol. 2, no. 10, pp. 685-694, 2001.

[2] J. R. Andrews-Hanna, J. S. Reidler, J. Sepulcre, R. Poulin, and R. L. Buckner, "Functional-anatomic fractionation of the brain's default network," Neuron, vol. 65, no. 4, pp. 550-562, 2010.

[3] W. W. Seeley, R. K. Crawford, J. Zhou, B. L. Miller, and M. D. Greicius, "Neurodegenerative diseases target large-scale 
human brain networks," Neuron, vol. 62, no. 1, pp. 42-52, 2009.

[4] R. L. Buckner, J. R. Andrews-Hanna, and D. L. Schacter, "The brain's default network: anatomy, function, and relevance to disease," Annals of the New York Academy of Sciences, vol. 1124, pp. 1-38, 2008.

[5] M. Wermke, C. Sorg, A. M. Wohlschläger, and A. Drzezga, "A new integrative model of cerebral activation, deactivation and default mode function in Alzheimer's disease," European Journal of Nuclear Medicine and Molecular Imaging, vol. 35, no. 1, pp. S12-S24, 2008.

[6] A. S. Fleisher, A. Sherzai, C. Taylor, J. B. S. Langbaum, K. Chen, and R. B. Buxton, "Resting-state BOLD networks versus task-associated functional MRI for distinguishing Alzheimer's disease risk groups," NeuroImage, vol. 47, no. 4, pp. 16781690, 2009.

[7] P. McCaffrey, T. Fagan, and E. Landhuis, "Alzheimer research series on the default network," Journal of Alzheimer's Disease, vol. 19, no. 2, pp. 747-758, 2010.

[8] D. Zhang and M. E. Raichle, "Disease and the brain's dark energy," Nature Reviews Neurology, vol. 6, no. 1, pp. 15-28, 2010.

[9] J. Shin, W. Tsui, Y. Li et al., "Resting-state glucose metabolism is associated with the pattern of amyloid pathology in alzheimer's disease," International Journal of Alzheimer's Disease, vol. 2011, Article ID 759780, 2011.

[10] S. E. Arnold, B. T. Hyman, J. Flory, A. R. Damasio, and G. W. Van Hoesen, "The topographical and neuroanatomical distribution of neurofibrillary tangles and neuritic plaques in the cerebral cortex of patients with alzheimer's disease," Cerebral Cortex, vol. 1, no. 1, pp. 103-116, 1991.

[11] H. Braak and E. Braak, "Neuropathological stageing of Alzheimer-related changes," Acta Neuropathologica, vol. 82, no. 4, pp. 239-259, 1991.

[12] S. Minoshima, B. Giordani, S. Berent, K. A. Frey, N. L. Foster, and D. E. Kuhl, "Metabolic reduction in the posterior cingulate cortex in very early Alzheimer's disease," Annals of Neurology, vol. 42, no. 1, pp. 85-94, 1997.

[13] V. Kepe, J. R. Barrio, S. C. Huang et al., "Serotonin 1A receptors in the living brain of Alzheimer's disease patients," Proceedings of the National Academy of Sciences of the United States of America, vol. 103, no. 3, pp. 702-707, 2006.

[14] K. Meguro, X. Blaizot, Y. Kondoh, C. Le Mestric, J. C. Baron, and C. Chavoix, "Neocortical and hippocampal glucose hypometabolism following neurotoxic lesions of the entorhinal and perirhinal cortices in the non-human primate as shown by PET. Implications for Alzheimer's disease," Brain, vol. 122, no. 8, pp. 1519-1531, 1999.

[15] K. Shoghi-Jadid, G. W. Small, E. D. Agdeppa et al., "Localization of neurofibrillary tangles and beta-amyloid plaques in the brains of living patients with alzheimer disease," American Journal of Geriatric Psychiatry, vol. 10, no. 1, pp. 24-35, 2002.

[16] G. W. Small, V. Kepe, L. M. Ercoli et al., "PET of brain amyloid and tau in mild cognitive impairment," New England Journal of Medicine, vol. 355, no. 25, pp. 2652-2663, 2006.

[17] P. M. Thompson, M. S. Mega, R. P. Woods et al., "Cortical change in Alzheimer's disease detected with a disease-specific population-based brain atlas," Cerebral Cortex, vol. 11, no. 1, pp. 1-16, 2001.

[18] J. L. Whitwell, S. A. Przybelski, S. D. Weigand et al., "3D maps from multiple MRI illustrate changing atrophy patterns as subjects progress from mild cognitive impairment to Alzheimer's disease," Brain, vol. 130, no. 7, pp. 1777-1786, 2007.
[19] J. R. Barrio, S. C. Huang, G. Cole et al., "Pet imaging of tangles and plaques in Alzheimer disease with a highly hydrophobic profile," Journal of Labelled Compounds and Radiopharmaceuticals, vol. 42, no. 1, pp. S194-S195, 1999.

[20] E. D. Agdeppa, V. Kepe, J. Liu et al., "Binding characteristics of radiofluorinated 6-dialkylamino-2-naphthylethylidene derivatives as positron emission tomography imaging probes for beta-amyloid plaques in Alzheimer's disease," The Journal of Neuroscience, vol. 21, no. 24, article RC189, 2001.

[21] R. L. Buckner, A. Z. Snyder, B. J. Shannon et al., "Molecular, structural, and functional characterization of Alzheimer's disease: evidence for a relationship between default activity, amyloid, and memory," Journal of Neuroscience, vol. 25, no. 34, pp. 7709-7717, 2005.

[22] C. Sorg, V. Riedl, M. Mühlau et al., "Selective changes of resting-state networks in individuals at risk for Alzheimer's disease," Proceedings of the National Academy of Sciences of the United States of America, vol. 104, no. 47, pp. 18760-18765, 2007.

[23] A. G. Vlassenko, S. N. Vaishnavi, L. Couture et al., "Spatial correlation between brain aerobic glycolysis and amyloid$\beta(\mathrm{A} \beta)$ deposition," Proceedings of the National Academy of Sciences of the United States of America, vol. 107, no. 41, pp. 17763-17767, 2010.

[24] J. Shin, S. Y. Lee, S. H. Kim, Y. B. Kim, and S. J. Cho, "Multitracer PET imaging of amyloid plaques and neurofibrillary tangles in Alzheimer's disease," NeuroImage, vol. 43, no. 2, pp. 236-244, 2008.

[25] J. Shin, S.-Y. Lee, S. J. Kim, S.-H. Kim, S.-J. Cho, and Y.B. Kim, "Voxel-based analysis of Alzheimer's disease PET imaging using a triplet of radiotracers: PIB, FDDNP, and FDG," NeuroImage, vol. 52, no. 2, pp. 488-496, 2010.

[26] J. Shin and S. Y. Lee, "2-(1-6-[(2-[Fluorine-18]fluoroethyl)(Methyl) amino]-2-naphthyl Ethylidene)malononitrile (FDDNP) positron emission tomography patterns in nondemented populations," American Journal of Geriatric Psychiatry, vol. 18, no. 2, p. 187, 2010.

[27] N. Tolboom, E. L. G. E. Koedam, J. M. Schott et al., "Dementia mimicking Alzheimer's disease Owing to a tau mutation: CSF and PET findings," Alzheimer Disease and Associated Disorders, vol. 24, no. 3, pp. 303-307, 2010.

[28] M. E. Raichle, A. M. MacLeod, A. Z. Snyder, W. J. Powers, D. A. Gusnard, and G. L. Shulman, "A default mode of brain function," Proceedings of the National Academy of Sciences of the United States of America, vol. 98, no. 2, pp. 676-682, 2001.

[29] D. A. Gusnard, E. Akbudak, G. L. Shulman, and M. E. Raichle, "Medial prefrontal cortex and self-referential mental activity: relation to a default mode of brain function," Proceedings of the National Academy of Sciences of the United States of America, vol. 98, no. 7, pp. 4259-4264, 2001.

[30] G. L. Shulman, J. A. Fiez, M. Corbetta et al., "Common blood flow changes across visual tasks-II. Decreases in cerebral cortex," Journal of Cognitive Neuroscience, vol. 9, no. 5, pp. 648-663, 1997.

[31] B. Mazoyer, L. Zago, E. Mellet et al., "Cortical networks for working memory and executive functions sustain the conscious resting state in man," Brain Research Bulletin, vol. 54, no. 3, pp. 287-298, 2001.

[32] K. A. McKiernan, J. N. Kaufman, J. Kucera-Thompson, and J. R. Binder, "A parametric manipulation of factors affecting task-induced deactivation in functional neuroimaging," Journal of Cognitive Neuroscience, vol. 15, no. 3, pp. 394-408, 2003. 
[33] M. D. Greicius, G. Srivastava, A. L. Reiss, and V. Menon, "Default-mode network activity distinguishes Alzheimer's disease from healthy aging: evidence from functional MRI," Proceedings of the National Academy of Sciences of the United States of America, vol. 101, no. 13, pp. 4637-4642, 2004.

[34] E. S. C. Korf, L. O. Wahlund, P. J. Visser, and P. Scheltens, "Medial temporal lobe atrophy on MRI predicts dementia in patients with mild cognitive impairment," Neurology, vol. 63, no. 1, pp. 94-100, 2004.

[35] G. B. Frisoni, N. C. Fox, C. R. Jack, P. Scheltens, and P. M. Thompson, "The clinical use of structural MRI in Alzheimer disease," Nature Reviews Neurology, vol. 6, no. 2, pp. 67-77, 2010.

[36] C. Lustig, A. Z. Snyder, M. Bhakta et al., "Functional deactivations: change with age and dementia of the Alzheimer type," Proceedings of the National Academy of Sciences of the United States of America, vol. 100, no. 2, pp. 14504-14509, 2003.

[37] K. A. Celone, V. D. Calhoun, B. C. Dickerson et al., "Alterations in memory networks in mild cognitive impairment and Alzheimer's disease: an independent component analysis," Journal of Neuroscience, vol. 26, no. 40, pp. 10222-10231, 2006.

[38] S. A. R. B. Rombouts, F. Barkhof, R. Goekoop, C. J. Stam, and P. Scheltens, "Altered resting state networks in mild cognitive impairment and mild Alzheimer's disease: an fMRI study," Human Brain Mapping, vol. 26, no. 4, pp. 231-239, 2005.

[39] K. Wang, M. Liang, L. Wang et al., "Altered functional connectivity in early Alzheimer's disease: a resting-state fMRI study," Human Brain Mapping, vol. 28, no. 10, pp. 967-978, 2007.

[40] W. E. Klunk, H. Engler, A. Nordberg et al., "Imaging brain amyloid in Alzheimer's disease with Pittsburgh compound-B," Annals of Neurology, vol. 55, no. 3, pp. 306-319, 2004.

[41] M. D. Ikonomovic, W. E. Klunk, E. E. Abrahamson et al., "Post-mortem correlates of in vivo PiB-PET amyloid imaging in a typical case of Alzheimer's disease," Brain, vol. 131, no. 6, pp. 1630-1645, 2008.

[42] J. L. Price, P. B. Davis, J. C. Morris, and D. L. White, "The distribution of tangles, plaques and related immunohistochemical markers in healthy aging and Alzheimer's disease," Neurobiology of Aging, vol. 12, no. 4, pp. 295-312, 1991.

[43] J. L. Price and J. C. Morris, "Tangles and plaques in nondemented aging and "preclinical" alzheimer's disease," Annals of Neurology, vol. 45, no. 3, pp. 358-368, 1999.

[44] M. E. Phelps and J. R. Barrio, "Correlation of brain amyloid with "aerobic glycolysis": a question of assumptions?" Proceedings of the National Academy of Sciences of the United States of America, vol. 107, no. 41, pp. 17459-17460, 2010.

[45] G. B. Cole, G. Keum, J. Liu et al., "Specific estrogen sulfotransferase (SULT1E1) substrates and molecular imaging probe candidates," Proceedings of the National Academy of Sciences of the United States of America, vol. 107, no. 14, pp. 6222-6227, 2010.

[46] M. A. Mintun, G. N. Larossa, Y. I. Sheline et al., "[11C]PIB in a nondemented population: potential antecedent marker of Alzheimer disease," Neurology, vol. 67, no. 3, pp. 446-452, 2006.

[47] H. Engler, A. Forsberg, O. Almkvist et al., "Two-year followup of amyloid deposition in patients with Alzheimer's disease," Brain, vol. 129, no. 11, pp. 2856-2866, 2006.

[48] C. R. Jack Jr., V. J. Lowe, and S. D. Weigand, “Alzheimer's Disease Neuroimaging Initiative. Serial PIB and MRI in normal, mild cognitive impairment and Alzheimer's disease: implications for sequence of pathological events in Alzheimer's disease," Brain, vol. 132, pp. 1355-1365, 2009.
[49] B. T. Hyman and T. Gomez-Isla, "Alzheimer's disease is a laminar, regional, and neural system specific disease, not a global brain disease," Neurobiology of Aging, vol. 15, no. 3, pp. 353-354, 1994.

[50] M. C. De Lacoste and C. L. White III, "The role of cortical connectivity in Alzheimer's disease pathogenesis: a review and model system," Neurobiology of Aging, vol. 14, no. 1, pp. 1-16, 1993.

[51] L. Berg, D. W. McKeel, J. P. Miller et al., "Clinicopathologic studies in cognitively healthy aging and Alzheimer disease: relation of histologic markers to dementia severity, age, sex, and apolipoprotein E genotype," Archives of Neurology, vol. 55, no. 3, pp. 326-335, 1998. 


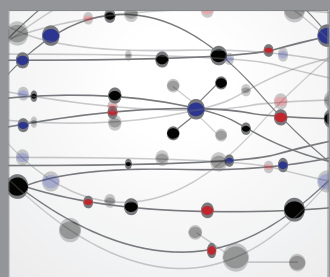

The Scientific World Journal
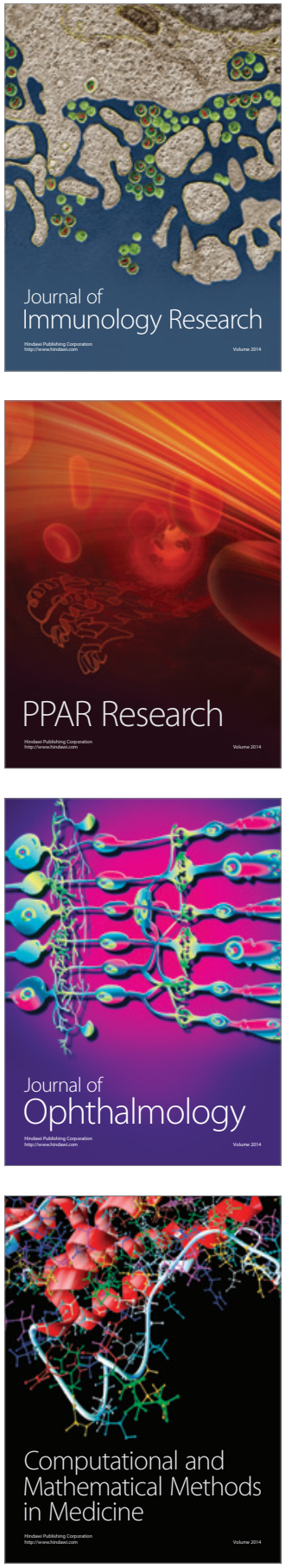

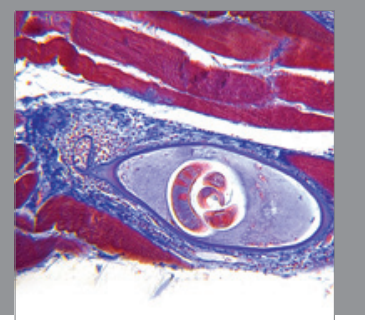

Gastroenterology

Research and Practice
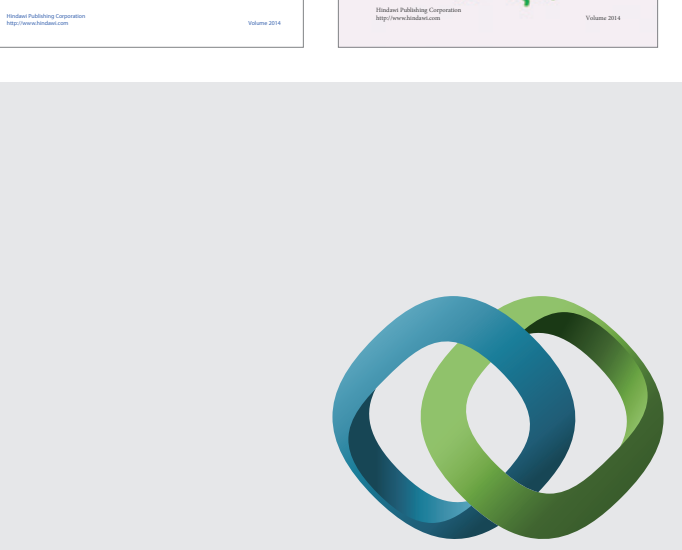

\section{Hindawi}

Submit your manuscripts at

http://www.hindawi.com
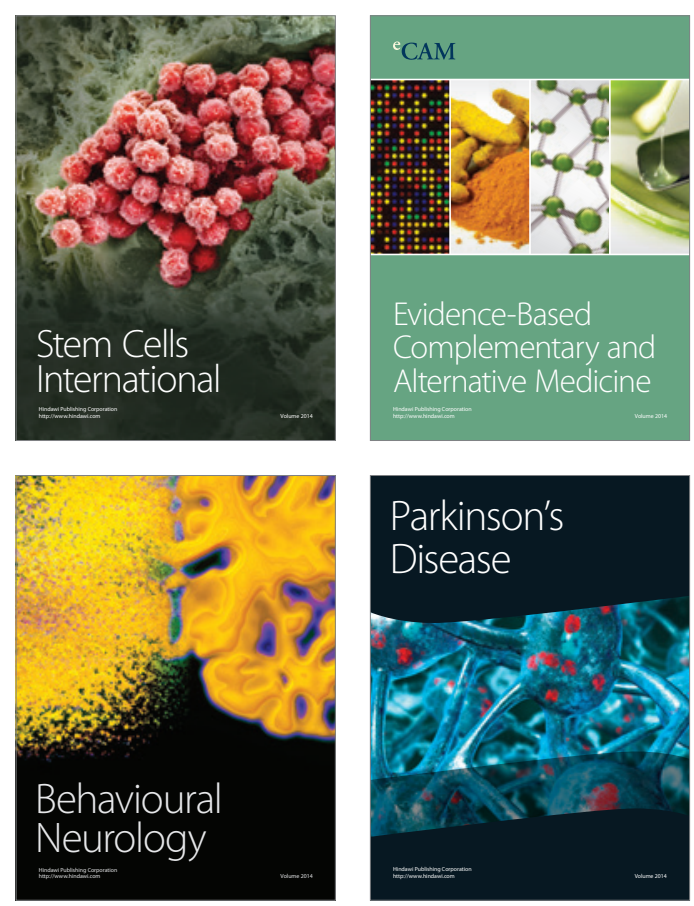

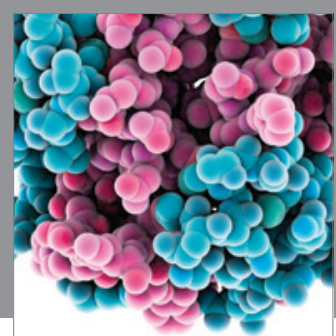

Journal of
Diabetes Research

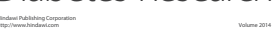

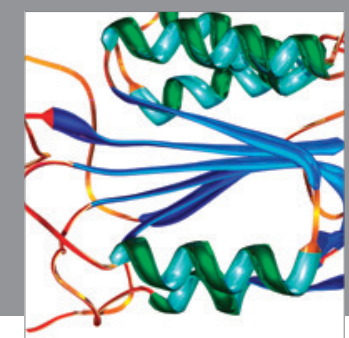

Disease Markers
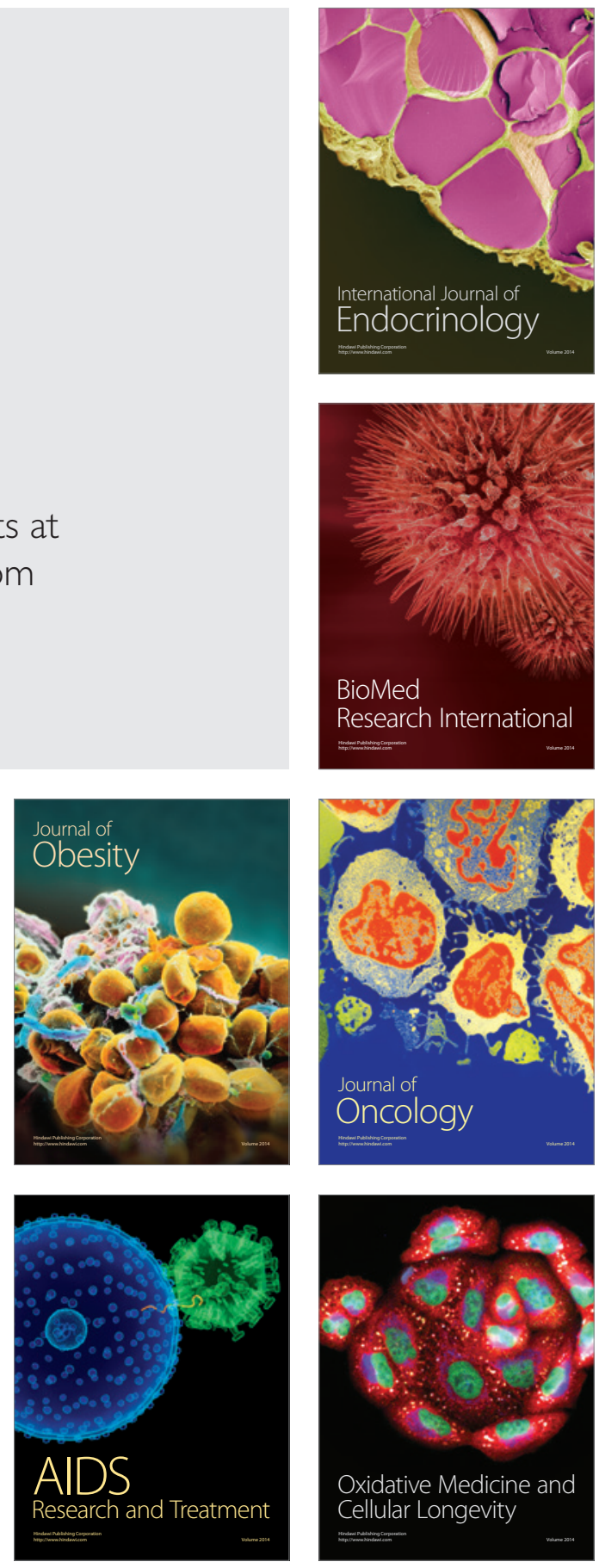